\title{
Optimization of Fermentation Conditions for Antibiotic Production by Actinomycetes YJ1 Strain against Sclerotinia sclerotiorum
}

\author{
Qin Song ${ }^{1}$, Yun Huang ${ }^{1} \&$ Hui Yang ${ }^{1}$ \\ ${ }^{1}$ Department of Plant Pathology, Sichuan Agricultural University, No. 211 Huimin Road of Wenjiang district, \\ Chengdu 611130, China \\ Correspondence: Yun Huang, Department of Plant Pathology, Sichuan Agricultural University, No. 211 Huimin \\ Road of Wenjiang district, Chengdu 611130, China. E-mail: 5787huangyun@sina.com.cn
}

Received: March 6, 2012 Accepted: March 27, 2012 Online Published: May 22, 2012

doi:10.5539/jas.v4n7p95 URL: http://dx.doi.org/10.5539/jas.v4n7p95

\begin{abstract}
In order to improve the productivity of activities substance of actinomycetes $\mathrm{YJ} 1$, the effects of the medium components, inoculation volume, medium capacity, rotary speed, fermentation time, temperature and initial $\mathrm{pH}$ on the biological activity of YJ1 were investigated by detecting the mycelium growth inhibition rate of Sclerotinia sclerotiorum. The results show that the inhibition rate is the highest under the optimum medium composition $(\mathrm{g} / \mathrm{L})$, containing sucrose 10.0, soluble starch 10.0, soybean meal 20.0, $\mathrm{K}_{2} \mathrm{HPO}_{4} 0.5, \mathrm{MgSO}_{4} 0.5$, $\mathrm{NaCl} 1.0$ and $\mathrm{FeSO}_{4}$ 0.01. The maximum antibiotic activity is obtained at the inoculation volume $5 \%$, medium volume in $250 \mathrm{ml}$ flask $75 \mathrm{ml}$, rotary speed of $180 \mathrm{r} / \mathrm{min}$, fermentation time 4 days, temperature $28^{\circ} \mathrm{C}$ and initial $\mathrm{pH}$ 7.0. The inhibition rate has been increased by $6.71 \%$ under the optimized condition.
\end{abstract}

Keywords: actinomycetes, fermentation, optimization, Sclerotinia sclerotiorum

\section{Introduction}

Exploring biological fungicides from microbial metabolites is an important research direction of fungicides and has been received more attention (NengGong, 2001). Currently, about 1000 varieties of bioactive substances from microorganisms have been discovered, and approximately $2 / 3$ of active substances come from actinomycetes, such as Novobiocin, Zhongshengmycin, etc (Xiao-hei Xing, 1999; Chang-xiong Zhu, 2002; Lei Zhao, 1998; Yue Feng Shi, 2004).

Actinomycetes are important soil microorganisms. They can produce different kinds of secondary metabolites and are the main strains of producing biological active substances. It has great application potential (Dong et al., 2003; Binwang, 2010). Most actinomycetes are heterotrophic and belong to the genus Streptomyces which is the largest proportion in the earth (Wei-yan Min et al., 2000; Hua Li, 2007). The mycelium of actinomycetes can elongate and branch unlimitedly. Therefore, actinomycetes mycelia fragment can grow into new mycelium in appropriate conditions by liquid shaking cultivation or industrial fermentation (Ruan-ji Sheng, 1990; Chen, et al., 1999; Elibol, 2002).

The levels of fermentation are related to fermentation time, ventilation, temperature, initial $\mathrm{pH}$ etc. Biocontrol strain fermentation is greatly influenced by the combination of media components and culture conditions in laboratory or industrial fermentation. Scientific and rational optimization of fermentation process not only can greatly improve the levels of eventual products, also reduce fermentation costs. Most actinomycetes have a higher requirement for oxygen to grow and optimally produce metabolites. However, medium contained a lot of organic and inorganic substances lead to a low level of dissolved oxygen, therefore, they need proper ventilation conditions to meet the requirements of dissolved oxygen (Qi-rui He, 2010). Fermentation time is a very important factor, which affect the yield and quality of metabolites. Fermentation temperature also affect the growth of actinomycetes. The optimum growth temperature is $23-37{ }^{\circ} \mathrm{C}$ for most actinomycetes (Breidt et al., 1995; Stal \& Moezelaar, 1997; Spyropoulou et al., 2001; Jian Chen, 2003). The optimum pH is 6.0 to 8.0 and the stable $\mathrm{pH}$ is maintained adding appropriate buffer (Lian-xiang Du, 1992).

Actinomycete strain YJ1 was isolated from branches of Ginkgo. It was identified as Streptomyces felleus based on morphological, physiological and biochemical characteristics, and the analysis of 16S rDNA sequence (Jia Yao, 2010). It had obvious effects by using the antagonistic actinomycetes to control S.sclerotiorum in laboratory, greenhouse and field trials (Jia Yao, 2010). But the suitable fermentation condition has not been reported. In this study, we tried to optimize fermentation condition of S. felleus YJ1 and determine the optimal combination of the 
medium composition and culture conditions to improve the active ingredient content, reduce costs, enhance the control effect and lay basis in real production application.

\section{Materials and Methods}

\subsection{Organism}

Streptomyces felleus YJ1 was isolated from branches of Ginkgo tree and stored in the laboratory. Sclerotinia sclerotiorum was isolated from sclerotia formated in the stem of incidence rape from Yaan, China (Wang \& Yao 2009).

\subsection{Culture Conditions and Medium}

Streptomyces felleus YJ1 was maintained on Gause's medium No.1 and S. sclerotiorum on PDA. They were stored at $4^{\circ} \mathrm{C}$ until required.

The medium compositions were as follows:

Gause's medium No.1 contained (g/L): soluble starch 20.0, $\mathrm{KNO}_{3} 1.0, \mathrm{NaCl} 0.5, \mathrm{~K}_{2} \mathrm{HPO}_{4} 0.5, \mathrm{MgSO}_{4} \cdot 7 \mathrm{H}_{2} \mathrm{O} 0.5$, $\mathrm{FeSO} 4 \cdot 7 \mathrm{H}_{2} \mathrm{O}$ 0.01, $\mathrm{pH} 7.2$.

A medium contained $(\mathrm{g} / \mathrm{L})$ : cornstarch 15.0 , glucose 10.0 , soybean meal 20.0, yeast paste $4.0, \mathrm{NaCl} 1, \mathrm{KH}_{2} \mathrm{PO}_{4} 1$, $\mathrm{CaCO}_{3} 3, \mathrm{pH}$ 7.2-7.4.

B medium contained (g/L): glucose 20.0, soybean meal 20.0, peptone 6, $\mathrm{NaCl} 5, \mathrm{CaCO}_{3} 4, \mathrm{pH}$ 7.2-7.4.

C medium contained $(\mathrm{g} / \mathrm{L})$ : cornstarch 30.0 , glucose 15.0, soluble starch 10.0 , soybean meal 20.0, $\mathrm{NH}_{4} \mathrm{Cl} 3.0$, $\mathrm{CaCO}_{3} 3.0, \mathrm{pH}$ 7.2-7.4.

D medium contained $(\mathrm{g} / \mathrm{L})$ : soluble starch 5.0, glucose 3.0, soybean meal 5.0, peptone 4.0, yeast paste $4.0, \mathrm{KNO}_{3}$ 1.0, $\mathrm{NaCl} 0.5, \mathrm{CaCO}_{3} 0.5, \mathrm{KH}_{2} \mathrm{PO}_{4} 0.5, \mathrm{MgSO}_{4} 0.5, \mathrm{FeSO}_{4} 0.01, \mathrm{pH}$ 7.2-7.4.

E medium contained $(\mathrm{g} / \mathrm{L})$ : millet 10.0, glucose 10.0, peptone 3.0, $\mathrm{NaCl}$ 2.0, $\mathrm{CaC}_{3}$ 2.0, $\mathrm{pH}$ 7.2-7.4.

F medium contained $(\mathrm{g} / \mathrm{L})$ : sucrose 10.0 , soluble starch 10.0, soybean meal 20.0, $\mathrm{K}_{2} \mathrm{HPO}_{4} \quad 0.5, \mathrm{MgSO}_{4} 0.5$, $\mathrm{NaCl}$ 1.0, $\mathrm{FeSO}_{4}$ 0.01, $\mathrm{pH}$ 7.2-7.4.

G medium contained (g/L): potato 200.0, glucose 20.0, $\mathrm{pH}$ 7.2-7.4.

\subsection{Batch Fermentation}

Stored strain of YJ1 was inoculated in Gause's medium No.1 and cultivated for 4 days at $28^{\circ} \mathrm{C}$ in incubator, then intercepted mycelium discs $(\mathrm{d}=5 \mathrm{~mm})$ and inoculated into terile medium in $250 \mathrm{~mL}$ flasks. The flasks were incubated in the dark at $28^{\circ} \mathrm{C}$ on an eberbach rotary shaker at $180 \mathrm{r} \cdot \mathrm{min}^{-1}$ for $7 \mathrm{~d}$. The cultures were centrifuged (8000r, $15 \mathrm{~min}, 4^{\circ} \mathrm{C}$ ) to separate the Actinomycetes cells and the supernatants. The supernatants were sterilized through $0.22 \mu \mathrm{m}$ bacterial filter and stored at $4^{\circ} \mathrm{C}$ until required.

\subsection{Antibiotic Activity Assay}

Antibiotic activity was measured by assaying the mycelium growth inhibition rate of $S$. sclerotiorum. Briefly, $1 \mathrm{~mL}$ sterile fermentation filtrate mixed with $9 \mathrm{~mL}$ PDA was replaced in a dish or without sterile fermentation filtrate as control. After the medium solidifying we inoculated $S$. sclerotiorum mycelium discs which as incubated for $3 \mathrm{~d}$ on the dish. The dishes were incubated in the dark at $28^{\circ} \mathrm{C}$ in incubator for $72 \mathrm{~h}$ and measured colony diameter of $S$. sclerotiorum using criss-cross method.

$$
\text { Inhibition rate }=\frac{\text { Colony diameter of treatment }- \text { Colony diameter of } \mathrm{CK}}{\text { Colony diameter of } \mathrm{CK}} \times 100 \%
$$

\subsection{Initial Antibiotic Activity Assay}

Stored strain of YJ1 fermented under the conditions as follows: medium D, inoculum volume $3 \%$, medium capacity $100 \mathrm{~mL}$, temperature $28^{\circ} \mathrm{C}$, shaking speed $180 \mathrm{r} \cdot \mathrm{min}^{-1}$, fermentation time $7 \mathrm{~d}$. Then the cultures were centrifuged and filtered to assay antibiotic activity.

\subsection{Experimental Design}

Previous studies indicated that medium, inoculation volume, aeration, time, temperature, agitation rate and initial pH had profound effects on production of antibiotics (Chen et al., 1996, Yang et al., 2001, 2006). Hence, univariate analysis and orthogonal design were used to find the optimized conditions for antibiotic production in flask fermentation experiments.

To determine the effect of medium, time, temperature, agitation rate and initial $\mathrm{pH}$ on the productivity of 
actinomycetes biocontrol metabolites, YJ1 was cultivated in different parameters as follows: medium (A, B, C, D, E, F and $\mathrm{G})$, time $(2,3,4,5,6,7,8,9$ and $10 \mathrm{~d})$, temperature $\left(20,24,28,32\right.$ and $\left.36^{\circ} \mathrm{C}\right)$, agitation rate $(60,100$, $140,180$ and $220 \mathrm{rmin}-1)$ and initial $\mathrm{pH}(5.0,5.5,6.0,6.5,7.0,7.5,8.0,8.5$ and 9.0) using univariate analysis, while inoculum volume $(1 \%, 3 \%, 5 \%$, and $7 \%)$ and medium capacity $(50,75,100$ and $150 \mathrm{~mL})$ using L16(4) orthogonal design (Table 1). The experiments were repeated three times.

Table 1. Experimental design of orthogonal design for optimization of inoculum volume and medium capacity

\begin{tabular}{ccc}
\hline Experiment & \multicolumn{2}{c}{ Coded value } \\
\cline { 2 - 3 } & $\mathrm{X}_{1}$ & $\mathrm{X}_{2}$ \\
\hline 1 & 1 & 1 \\
2 & 1 & 2 \\
3 & 1 & 3 \\
4 & 1 & 4 \\
5 & 2 & 1 \\
6 & 2 & 2 \\
7 & 2 & 3 \\
8 & 2 & 4 \\
9 & 3 & 1 \\
10 & 3 & 2 \\
11 & 3 & 3 \\
12 & 3 & 4 \\
13 & 4 & 1 \\
14 & 4 & 2 \\
15 & 4 & 3 \\
16 & 4 & 4 \\
\hline
\end{tabular}

Data in the table with the same letter means no significant difference

$\mathrm{X}_{1}$ inoculum volume $(1=1 \%, 2=3 \%, 3=5 \%, 4=7 \%)$

$\mathrm{X}_{2}$ medium capacity $(1=50,2=75,3=100,4=150)$

\section{Results}

\subsection{Initial Antibiotic Activity}

The colony of $S$. sclerotiorum on potato dextrose agar (PDA) with broth was smaller than control. The mycelia growth of $S$. sclerotiorum was significantly inhibited and the antibiotic rate of broth to S. Sclerotiorum was $83.98 \%$.

\subsection{Effect of Fermentation Medium}

In this study the effects of fermentation medium for actinomycetes producing biocontrol metabolites on the mycelial growth of S. sclerotiorum are presented in Figure 1. The results showed that the mycelial growth of S.sclerotiorum was inhibited. It indicated that YJ1 strains could produce antibiotic metabolites in different fermentation media. However, the production of $\mathrm{YJ} 1$ in $\mathrm{F}$ medium led to the maximum inhibition of the mycelium growth of $S$. Sclerotiorum. It was about $90.31 \%$. Therefore, F medium was good candidate for YJ1 to produce biocontrol metabolites among the seven fermentation media examined.

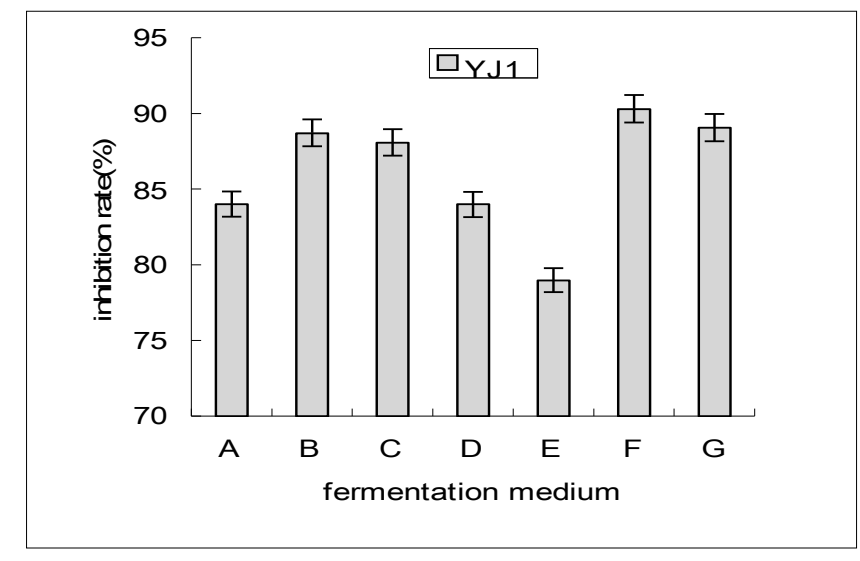

Figure 1. Effects of fermentation medium on the mycelial growth of S. sclerotiorum 


\subsection{Effect of Inoculation Volume and Medium Capacity}

Production of antibiotics by Streptomyces spp. is generally believed to be an aerobic process. Therefore, dissolved oxygen (DO) is an important factor in the fermentation of $S$. felleus YJ1. In shaken flasks, oxygen supply is related to medium volume. Otherwise, inoculation volume can affect the metabolites accumulation. A lower inoculum density may reduce product formation, whereas a higher inoculum may lead to the poor product formation, especially the large accumulation of toxic substances and also cause the reduction of dissolved oxygen (Mudgetti, 1986).

As seen in Figure 2, the inoculum size and medium capacity appeared to have visible effect on inhibiting the mycelial growth of S. sclerotiorum. When the inoculum volume and medium capacity were $5 \%$ and $75 \mathrm{~mL}$ respectively, the inhibition rate reached the maximum $(90.92 \%)$.

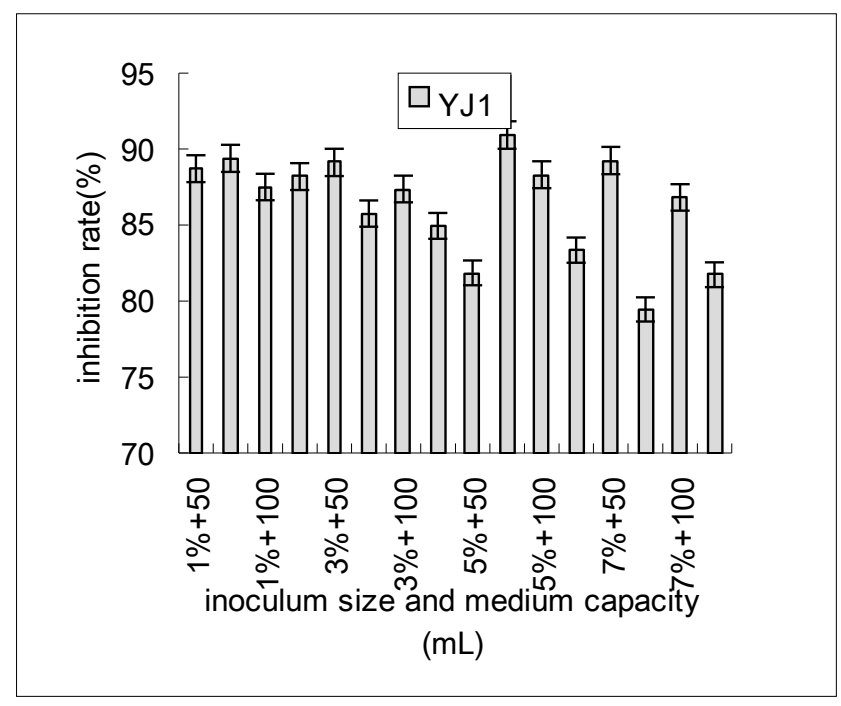

Figure 2. Effects of inoculum volume and medium capacity on the mycelial growth of S. sclerotiorum

\subsection{Effect of Fermentation Time}

Time is also an important factor to affect the fermentation. Increasing time does not mean producing more secondary metabolites. It may produce more toxins to inhibit the production of antimicrobial metabolites.

It can be seen in Figure 3, the fermentation time appeared to have visible effect on the mycelial growth inhibition of S.sclerotiorum in 2-4 days. The inhibition rate reached the maximum (90.07\%) at 4 days and then decreased slowly. However, there was no obvious difference in 4-10 days. The inhibition rate still remained about $86 \%$.

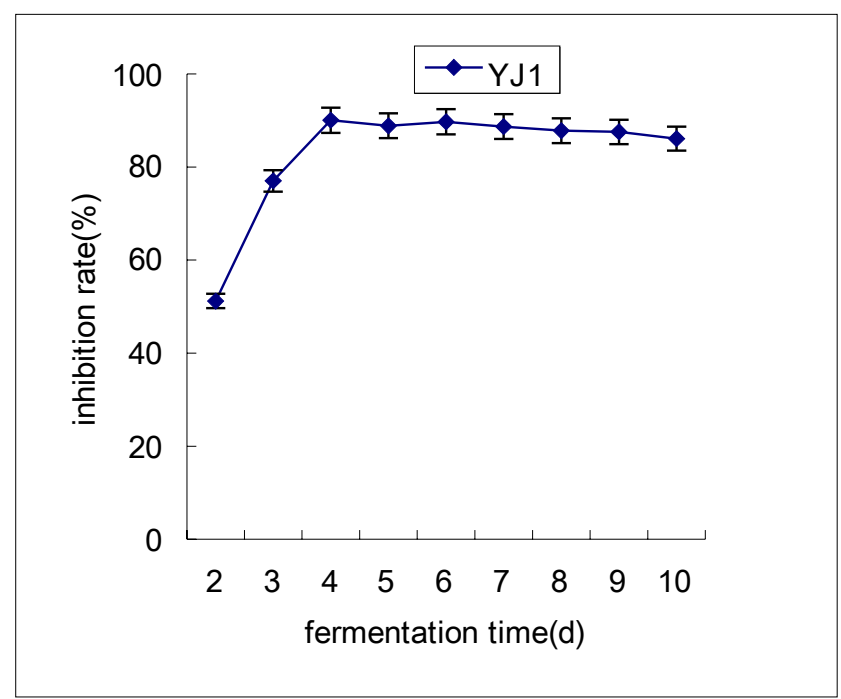

Figure 3. Effects of fermentation time on the mycelial growth of S.sclerotiorum 


\subsection{Effect of Fermentation Temperature}

To find out the optimal temperature for actinomycetes producing biocontrol metabolites to inhibit the mycelial growth of S. sclerotiorum, YJ1 was cultivated at various temperatures ranging from $20^{\circ} \mathrm{C}$ to $36^{\circ} \mathrm{C}$. As seen in Figure 4, YJ1 strains could produce biocontrol metabolites to inhibit the mycelial growth of S. sclerotiorum in 20-36. The inhibitory rate of the mycelial growth of S. sclerotiorum increased firstly and then decreased. It might be due to at higher or lower temperature than optimum, the growth of $\mathrm{YJ} 1$ was inhibited and further led to the biocontrol metabolites reduction. The maximum inhibitory rate reached $90.24 \%$ at $28^{\circ} \mathrm{C}$.

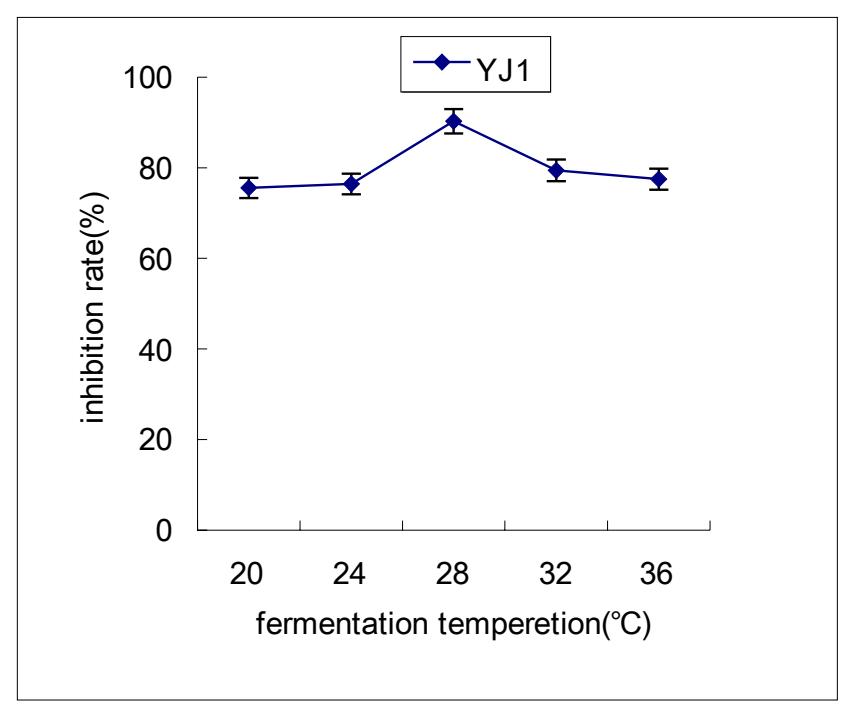

Figure 4. Effects of fermentation temperature on the mycelial growth of S.sclerotiorum

\subsection{Effect of Fermentation Agitation Rate}

Rotary speed can affect oxygen supply. As seen in Figure 5, the inhibition rate of S. sclerotiorum mycelial growth showed significant differences under the ranging from 60 to $220 \mathrm{r} \cdot \mathrm{min}^{-1}$. The inhibitory rate increased firstly and then decreased gradually upon increasing the agitation rate. When the speed was $180 \mathrm{r}$. $\mathrm{min}^{-1}$, the inhibitory rate reached its peak of about $90 \%$.

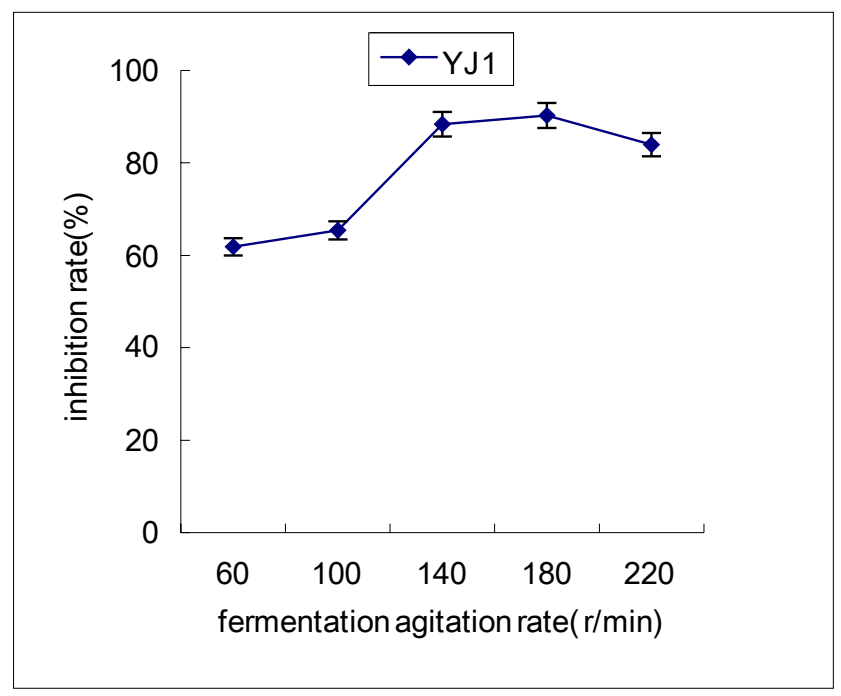

Figure 5. Effects of fermentation agitation rate on the mycelial growth of S. sclerotiorum 


\subsection{Effect of Initial pH of Fermentation Medium}

The effects of initial $\mathrm{pH}$ on the inhibitory rate of S. sclerotiorum are given in Figure 6. It can be seen that the initial $\mathrm{pH}$ of fermentation medium had a great impact on the antibacterial activity of YJ1. The antibacterial activity increased with increasing initial $\mathrm{pH}$ from 5.0 to 7.0, but any further increase in its values resulted in decreased antibacterial activity. It was due to too high or low initial $\mathrm{pH}$ would decrease the production of active substance which affected the inhibitory effect. Under acidic conditions it decreased significantly. When the initial $\mathrm{pH}$ was 7.0 , the maximum inhibitory rate reached $90.69 \%$. Therefore, the optimal initial $\mathrm{pH}$ was around 7.0 , which may relate to its natural growing environment.

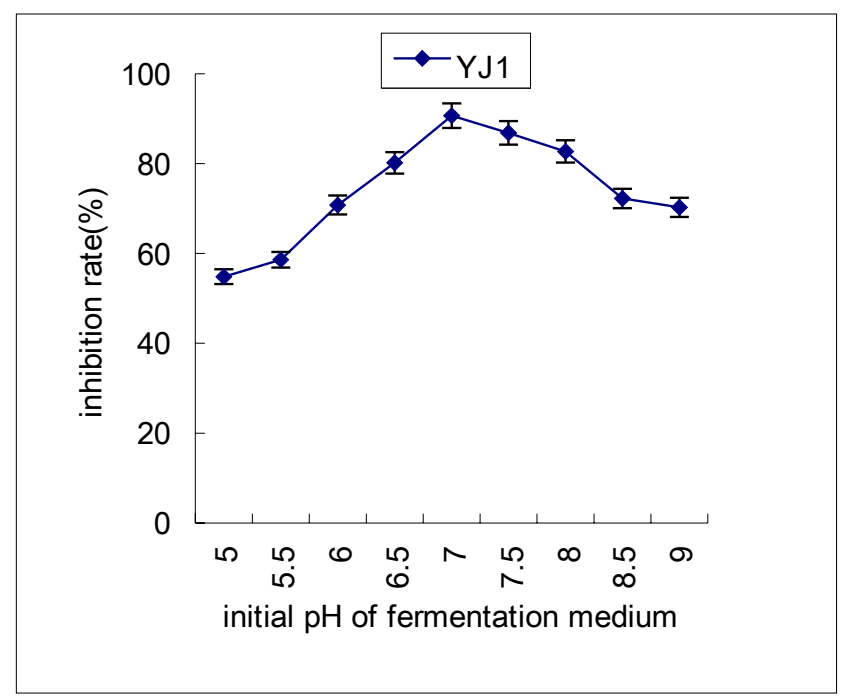

Figure 6. Effects of initial $\mathrm{pH}$ of fermentation medium on the mycelial growth of S.sclerotiorum

\section{Discussion}

Actinomycetes fermentation is a complex process, it not only depends on the performance and fermentation medium, also requires the suitable environmental conditions (such as inoculation volume, medium capacity, fermentation time, temperature, agitation rate and initial $\mathrm{pH}$ ). These factors may affect the antibiotics production. Fermentation has three obvious phases. Firstly, pre-fermentation is cell growth phase, nutrients are gradually consumed and began to produce antibiotics. Secondly, a large number of antibiotics are produced rapidly. Finally, in post-fermentation there is a slow accumulation of metabolites (Jin Wang, 2011). During this stage, actinomycetes even produce toxic metabolites. The reasons may due to cell senescence and autolysis, accumulation of final products or other toxic metabolites which strains can produce in a hostile environment, or the lack of precursors for synthesizing active substances (Liu et al., 2004). Therefore, the medium composition and ratio significantly affected fermentation process. Suitable nutrients can promote the synthesis of metabolites, cell growth, antibiotic fermentation unit, antibiotics extraction process, etc. (Kiers et al., 2000; Palmqvist \& Hahn-Hagerdal, 2000).

In this study, we focused on the optimization of nutritional constituents and culture conditions for production of antibiotics by Streptomyces felleus YJ1 through Single-factor analysis and orthogonal design to enhance the inhibitory rate of S. sclerotiorum. The approach allowed the determination of the culture conditions that gave the highest antibiotics activity for Streptomyces felleus YJ1. The better medium of strains YJ1 contained $(\mathrm{g} / \mathrm{L})$ : sucrose 10.0, soluble starch 10.0, soybean meal 20.0, $\mathrm{K}_{2} \mathrm{HPO}_{4} 0.5, \mathrm{MgSO}_{4} 0.5, \mathrm{NaCl} 1.0, \mathrm{FeSO}_{4} 0.01, \mathrm{pH}$ 7.2-7.4.

Ideal conditions for fermentation were inoculation volume $5 \%$, medium capacity $75 \mathrm{~mL}$, fermentation time $4 \mathrm{~d}$, temperature $28^{\circ} \mathrm{C}$, agitation rate $180 \mathrm{r}^{-m_{n}-1}$ and initial $\mathrm{pH}$ 7.0. In this study, the inhibitory rate had been increased by $6.71 \%$ under the optimized condition (Figure 7). The optimization of fermentation process should be considered not only the reduction of the costs of raw material but also the high antimicrobial activity. In this study the information obtained is useful for developing a Streptomyces felleus YJ1 cultivation process for efficient production of antibiotics on a large scale. 


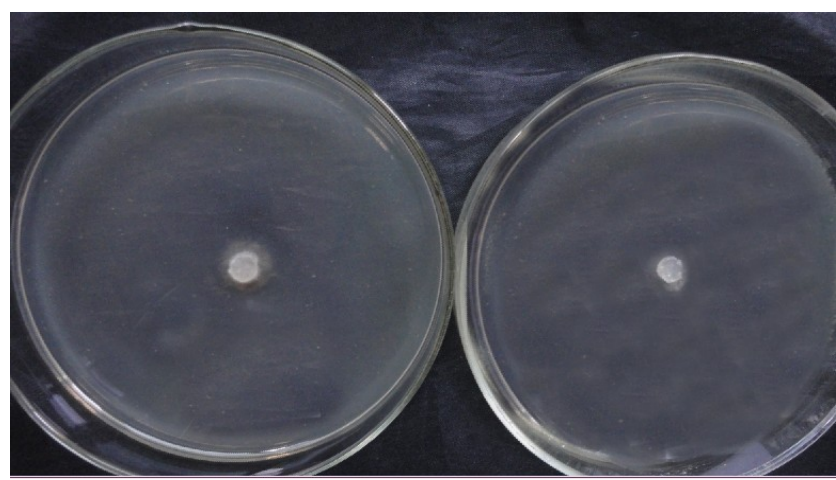

a

b

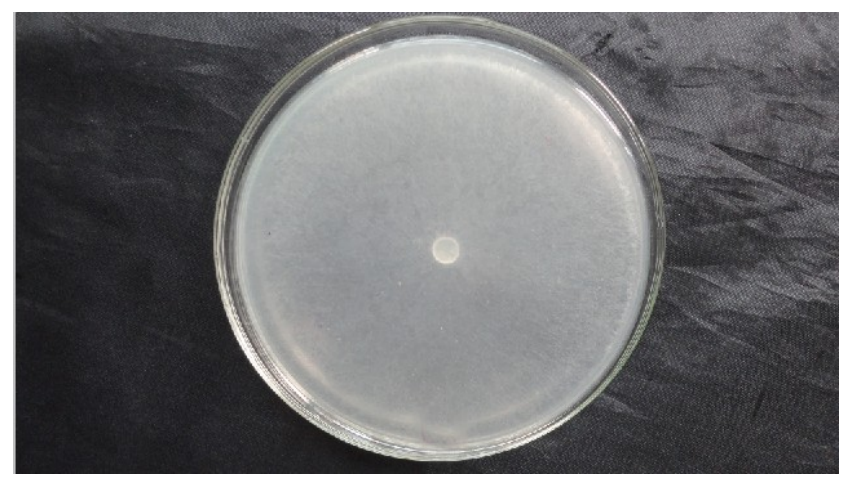

C

a: The PDA mixed with sterile fermentation filtrate which not optimized,

b: The PDA mixed with sterile fermentation filtrate which optimized,

c: control.

\section{Acknowledgements}

Figure 7. The colony size of S.sclerotiorum on the PDA

This work was supported by a Grant from Public service sectors (agriculture) research and special (nyhyzx07-054)" research and demonstration of technical solutions about S.sclerotiorum"

\section{References}

Bin, Wang. (2010). Study on isolation and screening of soil Actinomycetes Action to Botrytis Cinerea. Dissertation, Northwest Agriculture and Forestry University.

Breidt, F., Crowley, K. A., \& Fleming, H. P. (1995). Controlling cabbage fermentations with nisin and nisin-resistant Leuconostoc mesenteroides. Food Microbiol., 12, 109-116.

Chen, J., Du, G. C., \& Li, Y. (2003). Fermentation experimental techniques. Chemical Industry Press, Beijing.

Chen, Y., Krol, J., Sterkin, V. et al. (1999). New process control strategy used in a rapamycin fermentation. Process Biochem, 34, 383-389.

Dong, Y. M., Inigue, Z. A. L., Ahmer, B. M. M. et al. (2003). Kinetics and strain specificity of rhizosphere and endophytic colonization by enteric bacteria on seedlings of Medicago sativa and medicago truncatula. Appl. Environ. Microb., 69, 1783-1790.

Du, L. X. (1992). Industrial Microbiology experimental techniques. Tianjin.

Elibol, M. (2002). Response surface methodological approach for inclusion of perfluorocarbon in actinorhodin fermentation medium. Process Biochem., 38, 667-673.

Fan, H. M., Wang, B., Jiang, Y. et al. (2006). Study on Fermentation Condition of Antagonistic Actinomycetes XA-1. Journal of Anhui Agri., 34, 409-411, 418.

Gong, N. (2001). Biological pesticide development opportunities and challenges. Chin. J. Biotechnol., 17, 184-185. 
He, Q. R. (2010). Studies on Optimization of Fermentation Process of Streptomyces Lavendulae Xjy Strain and the Control Effect of Tomato Leaf Mould. Dissertation, Northwest Agriculture and Forestry University.

Kiers, J. L., Van laeken, A. E. A., Rombouts, F. M. et al. (2000). In vitro digestibility of Bacillus fermented soya bean. Int. J. Food Microbiol., 60, 163-169.

Liu, C. J., Duan, O. M., An, D. R. (2004). Antifungal actinomycetes screening and optimization of fermentation conditions. J. Microbiol., 24, 12-14.

Palmqvist, E., Hahn-Hagerdal, B. (2000). Fermentation of lignocellulosic hydrolysates II: inhibitors and mechanisms of inhibition. Bioresour. Technol., 74, 25-33.

Ruan, J. S. (1990). Present Application of Actinomycetes. Beijing.

Shi, Y. F., Sang, J. L., Zhu, L. H. et al. (2004). Studies on new microbical pesticide-streptomycin. Nuclear Agricultural Sciences, 18, 68-71.

Spyropoulou, K. E., Chorianopoulous, N. G., Skandamis, P. N. et al. (2001). Survival of Escherichia coli O157: H7 during the fermentation of Spanish-style green table olives (conservolea variety) supplemented with different carbon sources. Int. J. Food Microbiol., 66, 3-11.

Stal, L. J., \& Moezelaar, R. (1997). Fermentiaon in cyanobacteria. FEMS Microbiol. Rev., 21, 179-211.

Wang, J. (2011). Study on the Antagonistic Actinomycetes for Biocontrol of Plasmodiophora brassicae, Dissertation, Sichuan Agricultural University.

Wang, Y. H., Feng, J. T., Zhang, Q. et al. (2007). Optimization of fermentation condition for antibiotic production by Xenorhabdus nematophila with response surface methodology. J. Appl. Microbiol., 9, 47-48.

Wei, Y. M., Liu, D. Q., Tian, S. M. (2000). Streptomyces spp On the antagonism of several vegetable pathogens. Hebei Agricultural University, 23, 65-68.

Xing, X. H. (1999). Researches on molecular regulation of secondary metabolism of Actinomycetes. Biotechnol. $J ., 9,47-48$.

Xiong, Z. G. (2000). Fermentation Theory. Beijing

Xu, L. H., Li, W. J., Liu, Z. H. et al. (2007). Actinomycete systematics-Principles methods and practices. Beijing.

Yao, J. (2010). Study on the Biocontrol of Sclerotinia sclerotiorum by Antagonistic Actinomycetes. Dissertation, Sichuan Agricultural University.

Zhao, L., Song, J. H., Yang, H. T. et al. (1998). Preliminary studies on one agricultural Streptomyces spp. Chin. J. Biotechnol., 14, 18-20.

Zhu, C. X., Jiang, X. L., Shun, D. Y. et al. (2002). New agricultural antibiotics - Zhongshengmycin, 16, 14-17. 